\title{
Comparison of activation energies for the electrical conductivity of silicate glasses obtained by dc and ac techniques
}

\author{
E.C. Ziemath ${ }^{\text {a,* }}$, C.A. Escanhoela Jr ${ }^{\text {b }}$, M.L. Braunger ${ }^{c}$ \\ a Departamento de Física, IGCE, Universidade Estadual Paulista Júlio de Mesquita Filho (UNESP), CEP 13506-900 Rio Claro, SP, Brazil \\ b Laboratório Nacional de Luz Síncrotron (LNLS), CEP 13083-100 Campinas, SP, Brazil \\ c Departamento de Física Aplicada, IFGW, Universidade Estadual de Campinas (Unicamp), CEP 13083-859 Campinas, SP, Brazil
}

\section{A R T I C L E I N F O}

\section{Article history:}

Received 9 December 2016

Received in revised form 16 December 2016

Accepted 31 January 2017

Available online 7 February 2017

\section{Keywords:}

Silicate glasses

dc conductivity

Impedance spectroscopy

Activation energy

\begin{abstract}
A B S T R A C T
The electrical conductivity of glasses with composition (mol\%) $22 \mathrm{Na}_{2} \mathrm{O} \cdot 8 \mathrm{CaO} \cdot 65 \mathrm{SiO}_{2} \cdot 5 \mathrm{MO}_{2}(\mathrm{M}=\mathrm{Si}, \mathrm{Ti}, \mathrm{Ge}, \mathrm{Zr}$, $\mathrm{Sn}, \mathrm{Ce}$ ) was evaluated by two different experimental methods: low-voltage ac impedance and high-voltage dc resistivity. The ac measurements were carried out by the traditional impedance spectroscopy between 90 and $415^{\circ} \mathrm{C}$. In the dc method, a steady-state electric field of about $1 \mathrm{MV} / \mathrm{m}$ was applied during $5 \mathrm{~s}$ at temperatures between 100 and $210{ }^{\circ} \mathrm{C}$, and the onset current, $I_{\mathrm{o}}$, was measured. Activation energies between 0.71 and $0.81 \mathrm{eV}$ were obtained. The differences between the values obtained by the dc and the ac methods are within experimental errors. The high electric field does not influence the concentration nor the mobility of the charge carriers for these experimental conditions and glass compositions. The dc method applied showed to be a suitable alternative to determine the temperature dependence of the electric conductivity of glasses to further calculation of the activation energy.
\end{abstract}

C 2017 Elsevier B.V. All rights reserved.

\section{Introduction}

Electrical properties of dielectrics and materials with low charge carrier concentration had been measured by alternate current (ac) techniques, mainly the impedance spectroscopy, since the direct current (dc) techniques present inherent difficulties related to the sample polarization. Such polarization results in a fast decrease of the current immediately after the electric field had been turned on. For materials with low concentration of weakly bonded ions that can act as charge carriers or with permanent or induced electric dipoles, the dc current value is very low and it is therefore difficult to be measured accurately over longer time intervals. Soda-lime-silica glasses fall in such class of materials because the sodium ions, even at higher concentrations and more weakly bonded, have higher difficulty for long range displacements at near room temperatures. However, for some glass compositions, this situation can be overcome by applying high intensity electric fields at higher temperatures allowing that also higher current values can be measured. Nevertheless, the troubling aspect in dc procedures is that, with increasing time, the more mobile cations, like $\mathrm{Na}^{+}$, will be piled up at the

\footnotetext{
* Corresponding author.

E-mail address: ziemath@rc.unesp.br (E.C. Ziemath).
}

cathodic surface, while at the anodic region an alkali ion depleted layer is formed. The piled up cations can be reduced to metallic atoms taking electrons supplied by the cathode according to the reaction:

$\mathrm{Na}^{+}+\mathrm{e}^{-} \rightarrow \mathrm{Na}^{0}$

Such recombination process leads to the formation of a high intensity electric field in the thin layer between the $\mathrm{Na}^{+}$depleted layer and the anode while the electric field inside the glass sample is drastically reduced, leading to negligible currents. With the application of the ac techniques, this pile up and the depletion layer formation are avoided over large frequency and temperature ranges.

It has been observed that just after the application of high intensity steady-state electric fields on glass samples, a measurable onset current can be achieved [1]. With increasing temperature, this onset current also increases according to an Arrhenius behavior, allowing to calculate the activation energy for the electrical conductivity. In the present study, the activation energies for several soda-lime-silica glasses were obtained by a dc method and compared with those obtained by an ac method, i.e. impedance spectroscopy. According to the authors' knowledge, such kind of comparison has not been performed earlier for different glass samples with the same composition. 


\section{Theoretical approach}

\section{1. dc method}

The dc method applied in this study is the same as that used in thermal poling of glasses [1]. Fig. 1 shows a current curve for a soda-lime silica glass at $146{ }^{\circ} \mathrm{C}$ for an applied dc electric field of $1 \mathrm{MV} / \mathrm{m}$ [2]. In the present work, the important measurable parameter in this curve is the onset current, $I_{0}$, which takes place immediately after the electric field had been turned on. It is assumed to be measured before the formation of cationic pile up and depletion layers at the electrodes. Therefore, knowing the electrode area, $A$, the sample thickness, $d$, and the applied voltage between the electrodes, $V_{\mathrm{o}}$, the electrical conductivity at each temperature is:

$\sigma(T)=(d / A)\left(1 / V_{0}\right) I_{0}(T)$

The $I_{\mathrm{o}}$ values follow an Arrhenius behavior:

$I_{0}(T)=C \exp \left(-E_{\sigma}{ }^{\mathrm{dc}} / k_{\mathrm{B}} T\right)$

where $k_{\mathrm{B}}$ is the Boltzmann constant, $T$ is the temperature in $\mathrm{K}$ and $C$ is a constant. The activation energy for the electrical conductivity, $E_{\sigma}^{\mathrm{dc}}$, can then be determined by fitting the data in a $\log I_{\mathrm{o}}(T)$ vs. $T^{-1}$ plot by a straight line.

\section{2. ac method}

The most common ac technique employed to determine the electrical properties of materials is the impedance spectroscopy. It consists in applying a low amplitude ac voltage over a broad frequency range. In this technique the electrode polarization, common in dc measurements, is hindered for frequencies above certain value. The real and imaginary terms, $Z^{\prime}$ and $Z^{\prime \prime}$ resp., of the complex impedance, $Z^{*}$, are measured as a function of the frequency and temperature. These quantities are related by:

$$
Z^{*}=Z^{\prime}-j Z^{\prime \prime}
$$

The complex conductivity is:

$\sigma^{*}=\left(1 / Z^{*}\right)(d / A)=\sigma^{\prime}-j \sigma^{\prime \prime}$,

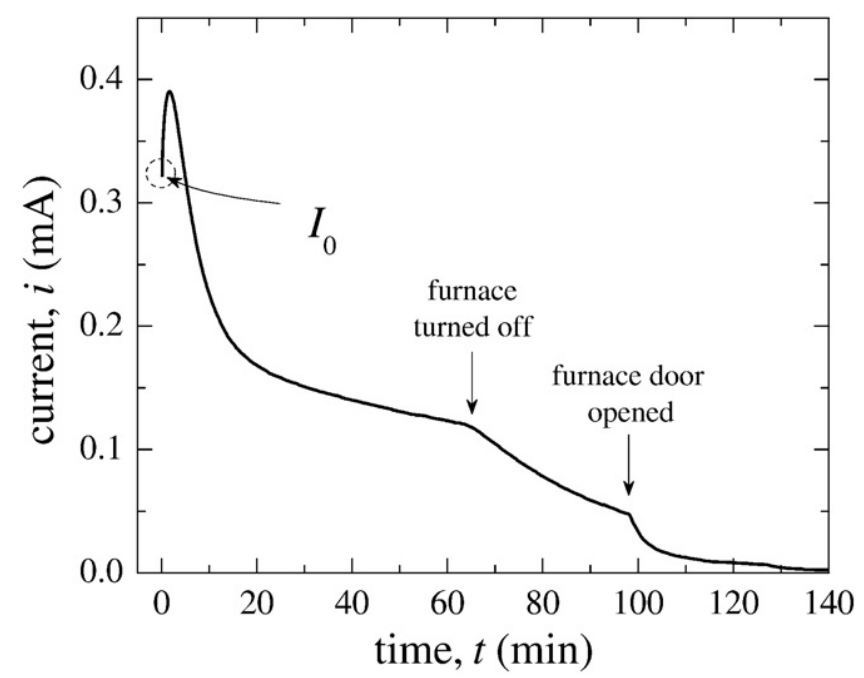

Fig. 1. Current curve for a commercial soda-lime-silica glass sample at $146{ }^{\circ} \mathrm{C}$ and applied electric field of $1 \mathrm{MV} / \mathrm{m}[2]$. where

$\sigma \prime=\left(Z \prime / Z^{2}\right)(d / A)$ and $\sigma^{\prime \prime}=\left(Z^{\prime \prime} / Z^{2}\right)(d / A)$.

The general behavior of $\sigma^{\prime}$ with the frequency $f$ is shown schematically in Fig. 2 . The dc regime occurs in the frequency range where the value of the electrical conductivity is constant, characterized by a plateau [3]. The dc electrical conductivity, $\sigma^{\prime}=\sigma_{\mathrm{dc}}$, is then calculated according to Eq. (6). The activation energy for the conductivity after this ac method, $E_{\sigma}^{\mathrm{ac}}$, is also obtained by an Arrhenius equation, analogous to Eq. (3):

$\sigma_{\mathrm{dc}}(T)=\sigma_{\mathrm{o}} \exp \left(-E_{\sigma}^{\mathrm{ac}} / k_{\mathrm{B}} T\right)$,

where $\sigma_{\mathrm{o}}$ is a pre-exponential factor, equivalent to the electrical conductivity of the sample at very high temperatures $(T \rightarrow \infty)$.

\section{Experimental procedures}

Glasses with composition (mol\%) $22 \mathrm{Na}_{2} \mathrm{O} \cdot 8 \mathrm{CaO} \cdot 65 \mathrm{SiO}_{2} \cdot 5 \mathrm{MO}_{2}$, $\mathrm{M}=\mathrm{Si}$, Ti, Ge, Zr, Sn, Ce, were obtained by melting appropriate quantities of sodium and calcium carbonates, quartz sand, and oxides of the other tetravalent cations, as previously reported [4]. The melting was performed in a platinum crucible at about $1450{ }^{\circ} \mathrm{C}$ for $2 \mathrm{~h}$, in an electric furnace. The melt was poured on stainless steel plate, pressed with another steel plate, quenched in cold water, crushed, and remelted. This protocol was repeated up to 4 times to ensure chemical homogeneity of the glass. After the last pour, the still-hot glass was transferred to a preheated furnace at $600{ }^{\circ} \mathrm{C}$ for annealing. This temperature is slightly higher than the glass transition temperatures, $T_{\mathrm{g}}$, of the glasses, which are below $580{ }^{\circ} \mathrm{C}$ [5]. The annealing furnace was further turned off to allow the glass to cool down slowly to room temperature in order to avoid internal stresses. Transparent and homogeneous glasses were obtained. As observed earlier [4], these glass compositions offer high resistance to devitrification. Samples were cut with a diamond low speed saw, lapped with $\mathrm{SiC}$ and polished with ceria-based glass polishing powder.

For the high-voltage measurements, samples had thickness between 1.2 and $1.6 \mathrm{~mm}$. The experimental setup is shown schematically in Fig. 3. The sample was placed between $10 \mathrm{~mm}$ diameter gold foils, gently pressed by stainless steel electrodes and accommodated in an electric furnace. The electrodes were connected to a high dc voltage power supply (Keithley, mod. 246). The applied voltage was between 1.2 and

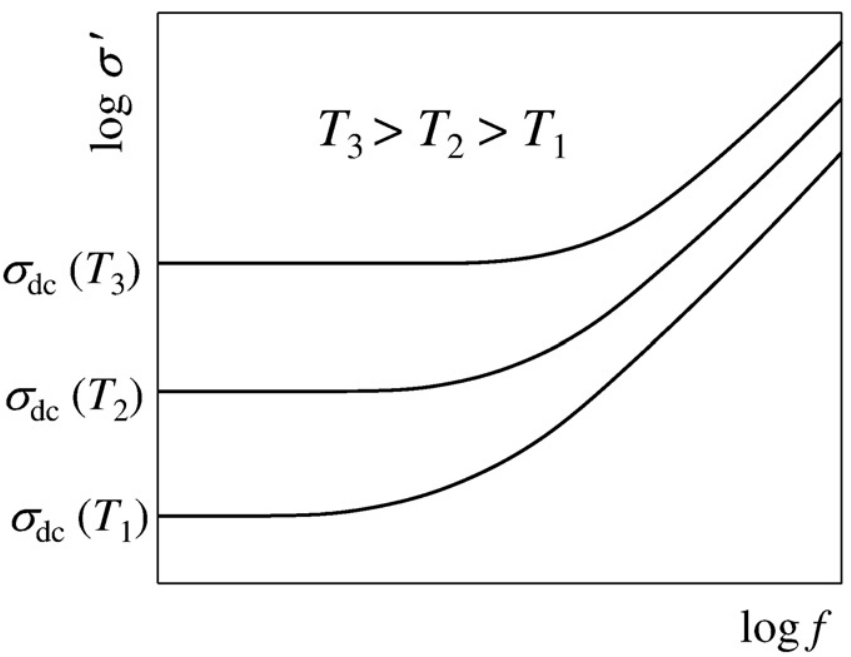

Fig. 2. Schematic behavior of the $\sigma^{\prime}$ vs. $f$ plot (di-log scales) at three different temperatures. 


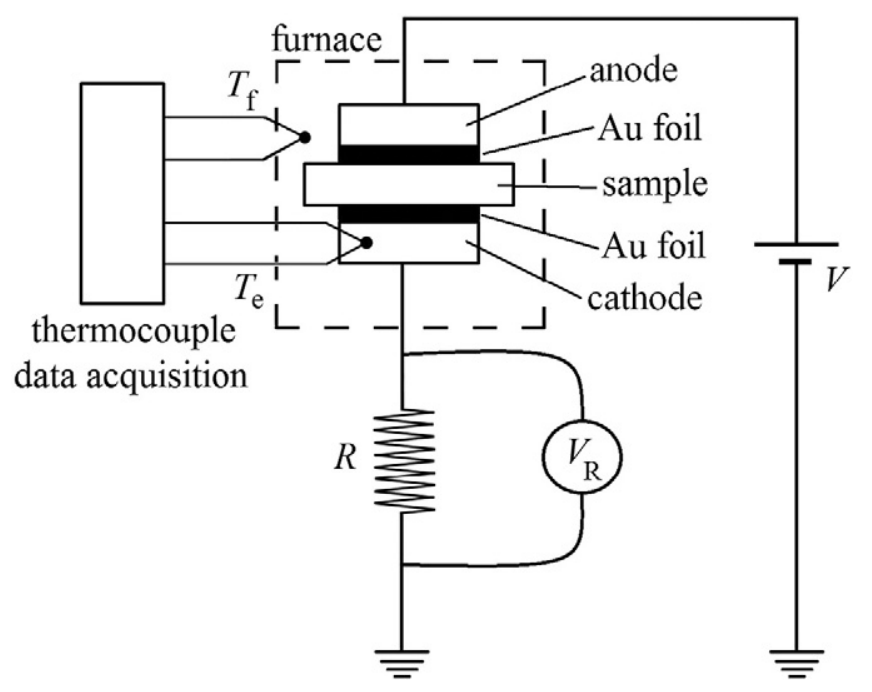

Fig. 3. Experimental setup for measuring the onset currents, $I_{0}$, by the dc method.

$1.6 \mathrm{kV}$ in order to generate an electric field of $1 \mathrm{MV} / \mathrm{m}$ on the sample. This field intensity was chosen in order to avoid deviation from linearity that takes place for very high fields [6,7]. Therefore, it was essential to choose an internal field intensity $E \ll k_{\mathrm{B}} T / q a$, were $a$ is the jump distance of a carrier with charge $q$. For $T=490 \mathrm{~K}\left(\sim 220^{\circ} \mathrm{C}\right)$ and $a \cong 4.5 \AA$ [4], one obtains that $E$ should be less than $95 \mathrm{MV} / \mathrm{m}$. The dielectric constant, $\kappa$, of the glasses is estimated to be higher than 30 and it increases with increasing temperature $[8,9]$, resulting in an internal field, $E=E_{\mathrm{o}} / \kappa$, lower than $0.3 \mathrm{MV} / \mathrm{m}$ for $E_{\mathrm{o}}=1 \mathrm{MV} / \mathrm{m}$. Hence the above condition is satisfied.

The field of $1 \mathrm{MV} / \mathrm{m}$ was applied to the sample during about $5 \mathrm{~s}$ at different temperatures. The voltage drop, $V_{R}$, on a resistor, $R=$ $(0.991 \pm 0.001) \mathrm{k} \Omega$, in series with the sample (Fig. 3$)$, was measured with a digital multimeter (Minipa-APPA, mod. ET-2609) with a data acquisition time of $0.5 \mathrm{~s}$. This voltage was measured simultaneously with the temperature of the sample by suitable hardware and software [2]. The electric current through the sample, $i(T)$, was calculated by $V_{\mathrm{R}} / R$. The $I_{0}$ values were determined experimentally at different temperatures and the conductivity for this particular situation was calculated by Eq. (2). At each new selected temperature, the high voltage was turned on only after the temperature of the sample had been stabilized. The time needed to achieve this stabilization was of about $90 \mathrm{~min}$. The $I_{0}$ values were obtained at temperatures between 100 and $220^{\circ} \mathrm{C}$.

The ac measurements were performed employing a Solartron Impedance Analyzer (mod. 1260A). The device was also interfaced with

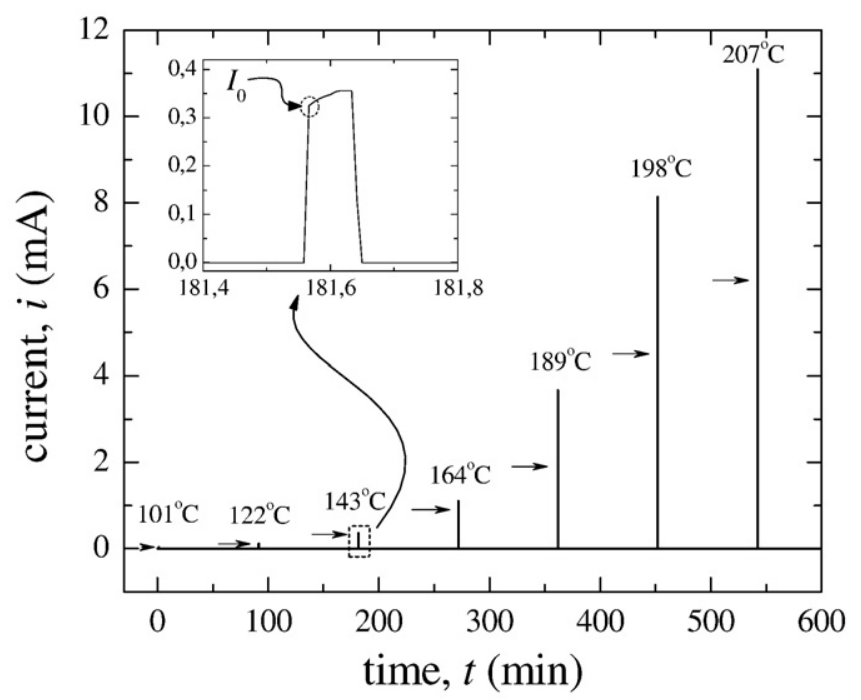

Fig. 5. Current pulses with about $5 \mathrm{~s}$ duration due to an external electric field of $1.0 \mathrm{MV} / \mathrm{m}$ on a Sn-containing glass at increasing temperature. The horizontal arrows indicate the onset current, $I_{0}$, for the corresponding temperatures. Inset: Magnified current pulse for $143^{\circ} \mathrm{C}$ and its $I_{\mathrm{O}}$.

a personal computer through suitable hardware and software. An applied voltage with amplitude of $2.0 \mathrm{~V}$ gave the less noisy results. The $Z$ ' and $Z^{\prime \prime}$ values where measured on $1 \mathrm{~mm}$ thick samples. Circular gold electrodes with diameters of $10 \mathrm{~mm}$ were evaporated on the opposite parallel surfaces. The frequency range between $1 \mathrm{~Hz}$ and $1 \mathrm{MHz}$ was explored at temperatures between 90 and $415^{\circ} \mathrm{C}$. Plots of $\log \sigma^{\prime}$ vs. $\log f$ were analyzed as mentioned in Section 2. Details of these procedures are presented elsewhere [4].

\section{Results}

Fig. 4(a) shows the behavior of the current pulses in a Si-containing glass sample at $145^{\circ} \mathrm{C}$ for applied voltage, $V_{\mathrm{o}}$, between 0.5 and $2.8 \mathrm{kV}$. Fig. 4(b) shows that the relation between $I_{0}$ and $V_{o}$ is linear up to $2.5 \mathrm{kV}$, indicating that electrode polarization is indeed negligible under the conditions stated above. Deviation from linearity is observed above $2.5 \mathrm{kV}$, which corresponds to an applied electric field of $1.7 \mathrm{MV} / \mathrm{m}$. Therefore, the chosen value of $1 \mathrm{MV} / \mathrm{m}$ for the applied electric field is within the linear domain. For voltages lower than $0.4 \mathrm{kV}$, electrical current could not be detected.

The data between 0.5 and $2.0 \mathrm{kV}$ in Fig. 4(b) were fitted by a straight line: $I_{\mathrm{o}}(\mathrm{mA})=-(90 \pm 2) \times 10^{-3}+(0.324 \pm 0.002) V_{\mathrm{o}}(\mathrm{kV})$. This leads
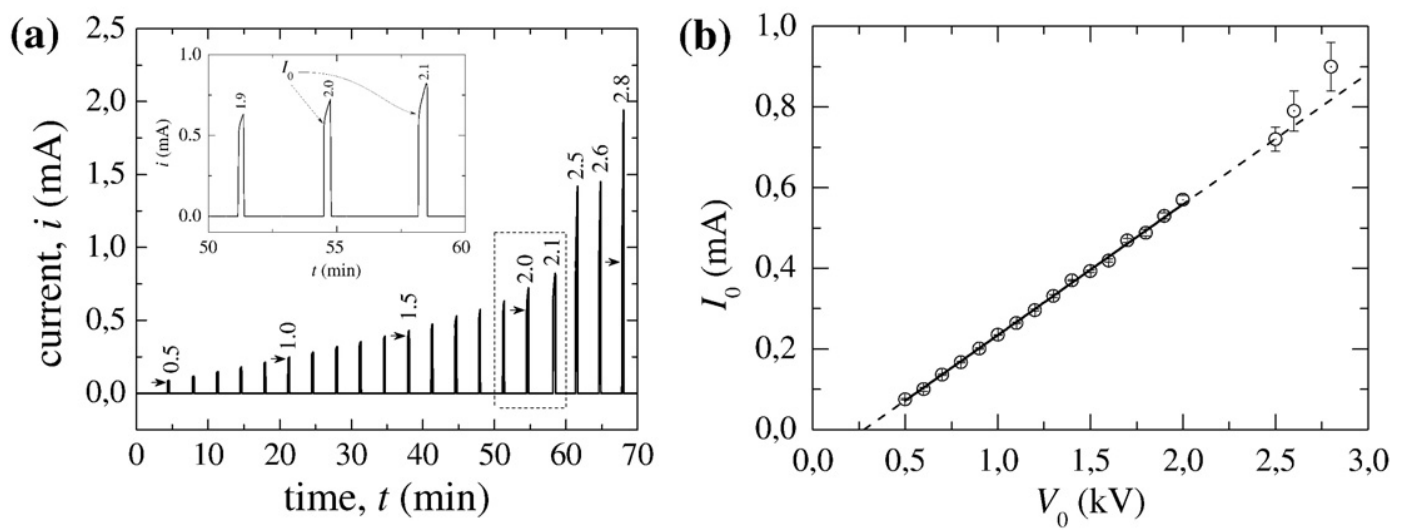

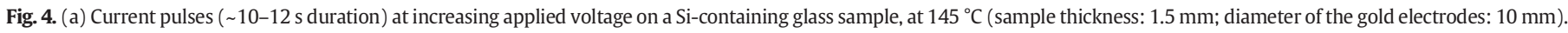

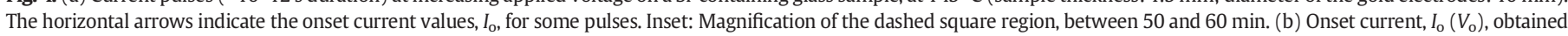
from (a); the solid line is the linear least square fit of the values between 0.5 and $2.0 \mathrm{kV}$ and the dashed lines are extrapolations. 


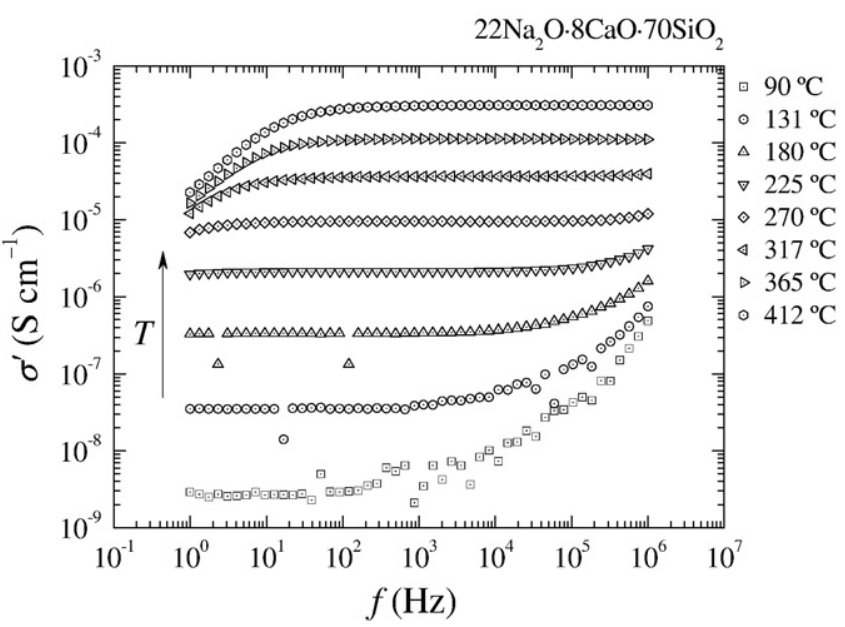

Fig. 6. $\sigma^{\prime}$ vs. $f$ plots for the Si-containing glass, from impedance spectroscopy measurements.

to a conductivity of $(6.2 \pm 0.9) \times 10^{-8} \mathrm{~S} \mathrm{~cm}^{-1}$, which agrees with the value obtained by ac measurements at nearly the same temperature: $\sim 6,7 \times 10^{-8} \mathrm{~S} \mathrm{~cm}^{-1}$.

Fig. 5 shows the results of the current pulses for a Sn-containing glass sample at increasing temperatures for $E_{0}=1.0 \mathrm{MV} / \mathrm{m}$. The values of $I_{\mathrm{o}}(T)$ were determined after enlarging the time scale, as shown in the inset. The time interval during which the high voltage had been held on was short enough so that the $I_{\mathrm{o}}$ values obtained at the following higher temperatures were not significantly affected by eventual previously formed depletion layer and pile up of cations at opposed electrodes. In the inset of Fig. 5, an increase of the current during the pulse interval can be observed. It is attributed to the Joule heating [1,7]. With increasing temperature, the initial slope of each current pulse also increases faster, leading to higher uncertainties in the determination of the $I_{\mathrm{o}}$ values. Similar measurements were performed on the other glass samples. Once knowing the values of $I_{\mathrm{o}}$ at several temperatures, the electrical conductivity was calculated by Eq. (2).

Fig. 6 presents a di-log plot of $\sigma^{\prime}$ vs. $f$ for the Si-containing glass, obtained from impedance measurements. The $\sigma^{\prime}$ values were calculated according to Eq. (6). The $\sigma^{\prime}=\sigma_{\mathrm{dc}}$ plateau shifts to higher frequencies with increasing temperature. Similar behavior was observed for all glasses in the present work. The decrease of $\sigma^{\prime}$ with reduction of the frequency at higher temperatures is due to electrode polarization, where charge carriers become trapped or cannot undergo electron-cation recombination at the cathode (Eq. (1)) [3,10].
Fig. 7 presents the Arrhenius plots of the conductivity for the Si- and Ge-containing glasses obtained by both methods. The activation energies, $E_{\sigma}^{\mathrm{ac}}$, and the pre-factor values, $\sigma_{\mathrm{o}}$, in Eq. (7), were calculated by the least-square analysis of these plots and the results for $E_{\sigma}^{\mathrm{ac}}$ are presented in Table 1. Fig. 8 is a bar plot of these values. The results obtained for each glass by both methods are in good agreement.

Fig. 7 shows also that the temperature range for the dc method is shorter than that for the ac method. The main limitation in the present dc experimental setup is that the electrical insulation becomes less efficient at higher temperatures and for very high electric fields. Additionally, at higher temperatures the current increases very fast, making it more difficult to determine $I_{\mathrm{o}}$ with enough accuracy.

\section{Discussion}

According to Fig. 8, the activation energy values obtained by the dc method are somewhat lower than those obtained by the ac method. The higher differences were observed for the $\mathrm{Ge}$ - and $\mathrm{Zr}$-containing glasses but they are lower than $5 \%$, and can be considered within the experimental uncertainties. The uncertainty of the measured $I_{0}$ at low temperatures is less than $7 \%$ and reaches $15 \%$ at higher temperatures. In spite of the high percentage errors of the $I_{0}$ values, the absolute values of the calculated conductivity are very close to those obtained by impedance spectroscopy, as can be observed in Fig. 7.

The cause of higher uncertainty of $I_{\mathrm{o}}$ maybe attributed to the measuring process. This current is calculated from values of $V_{R}$ measured on the series resistor (Fig. 3 ) at each $0.5 \mathrm{~s}$ of acquisition. It is determined by extrapolating the current curve into the time interval between two successive data acquisition, i.e., between $V=0$ up to $V_{\mathrm{o}}=(1 \mathrm{MV} / \mathrm{m})$ $d$. For higher temperatures, where the Joule effect is enhanced, the slope of the current curve immediately after the electric field had been turned on is too high, preventing a more accurate determination of this current. At lower temperatures, the values obtained by the dc and ac methods show a better agreement because the change in the dc current with the time is less pronounced (inset of Fig. 5), allowing a better accuracy of the $I_{\mathrm{o}}$ values.

The time interval between successive acquisitions can influence the measured values in specific situations because it is related to the time scale in which some phenomena take place inside the sample. Generally, the polarization of soda-lime glasses occurs at higher temperatures and long time scales (low frequencies) due to the formation of space charges at the sample surfaces close to the blocking electrodes. As a consequence, conductivity is reduced. According to Fig. 6, a reduction of the conductivity is more pronounced for temperatures higher than $250{ }^{\circ} \mathrm{C}$ and frequencies lower than $10 \mathrm{~Hz}$, i.e. for time scales higher than $0.1 \mathrm{~s}$. Analogous behavior had been observed for all glasses presently studied.

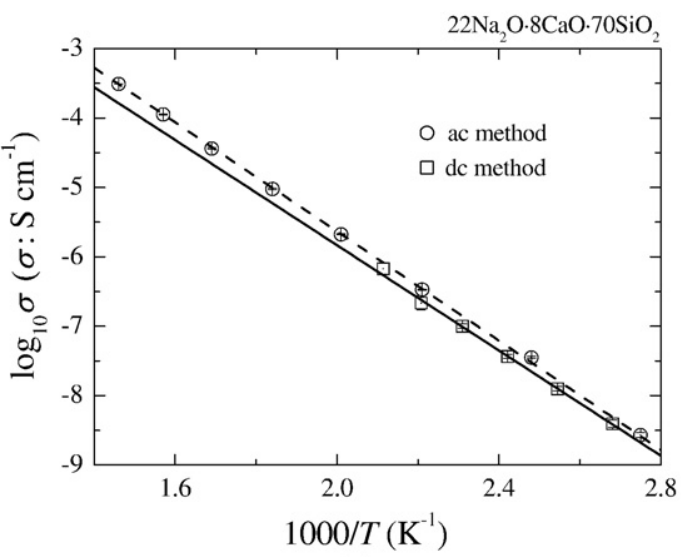

(a)

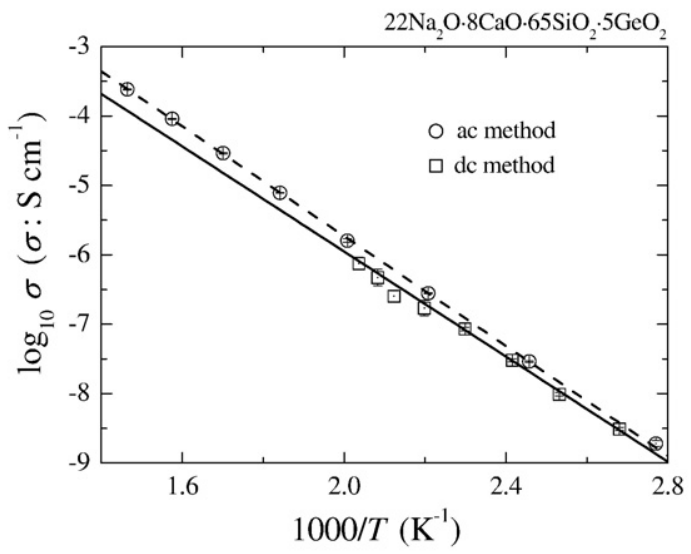

(b)

Fig. 7. Superposition of Arrhenius plots of the electrical conductivities obtained by the dc (dashed lines) and the ac (continuous lines) methods: (a) Si- and (b) Ge-containing glasses. 
The frequency associated to the acquisition time interval is $2 \mathrm{~Hz}$ and the $I_{\mathrm{o}}$ values were determined for temperatures lower than $220^{\circ} \mathrm{C}$. In such experimental conditions, the time scale of data acquisition is shorter than that for the buildup of space charge.

The electrical current measured in the dc method is due to the interaction of charges in the sample with the blocking electrodes. Characteristic phenomena related to this current take place immediately after the $V_{\mathrm{o}}$ has been turned on. Firstly, the electrodes are charged and the current increase very fast. The free charge, $q_{\mathrm{f}}$, at the electrodes induces bound charges, $q_{\mathrm{b}}$, on the sample surface, close to the electrodes. Thus, the sample becomes polarized due to electronic, atomic and dipolar polarizations of the sample which are processes that takes place at short time scales, corresponding to frequencies higher than $10^{6} \mathrm{~Hz}$ [11]. At larger time scales, i.e., for frequencies between $10^{6}$ and $1 \mathrm{~Hz}$, the more mobile cations $\left(\mathrm{Na}^{+}\right)$are now able to displace over larger distances, in a hopping process. In the ac measurements, this is characterized by the "plateaus" observed in Fig. 6, where the conductivity, $\sigma^{\prime}$, is constant for a broad frequency range. With increasing temperature and at higher time scales, an increasing concentration of cations move toward the cathode and away from the anode, therefore increasing the bond charge density, $\sigma_{\mathrm{b}}$, and also the polarization, $P_{\mathrm{b}}$, of the sample. The change of this polarization with the time is related to the measured current:

$i_{\mathrm{p}}(t)=\mathrm{d} q_{\mathrm{b}} / \mathrm{d} t=A\left(\mathrm{~d} P_{\mathrm{b}} / \mathrm{d} t\right)$,

where $A$ is the electrode area. The polarization behavior after the voltage had been turned on is strongly temperature dependent since Joule heating plays an increasing role at higher temperatures [1]. However, the onset current, $I_{0}$, measured within the $0.5 \mathrm{~s}$ interval and below $210^{\circ} \mathrm{C}$, is assumed to has not been influenced by such heating. Once the subsequent measured current is time dependent, the conductivity is also.

According to the classical theory, the electrical conductivity is given by $[3,12]$ :

$\sigma=n q \mu$,

where $n$ is the effective charge carrier concentration with charge $q$ and mobility $\mu$. According to Fig. 7, the conductivity of silicate glasses is temperature dependent. Then, $n$ or/and $\mu$ should also be temperature dependent according to $[13,14]$ :

$n(T)=n_{0} \exp \left(-E_{\mathrm{f}} / k_{\mathrm{B}} T\right)$

$\mu(T)=\mu_{\mathrm{o}} \exp \left(\mp E_{\mathrm{m}} / k_{\mathrm{B}} T\right)$,

where $n_{\mathrm{o}}$ is the concentration of the alkali cations in the glass composition and $E_{\mathrm{f}}$ and $E_{\mathrm{m}}$ are, resp., the energy required for the formation of a mobile ion and the migration energy of that ion. The minus/plus sign in the exponential of Eq. (11) means that the mobility increases/decreases with increasing temperature. For alkali silicate glasses, usually the minus sign prevails [15]. In this case, $E_{\sigma}=E_{\mathrm{f}}+E_{\mathrm{m}}$.

The reaction of the cations at the cathode according to Eq. (1), is considered to be relevant only at high temperatures and at time scales

Table 1

Activation energies for the electrical conductivity of the M-containing glasses obtained by the dc and ac methods. The $\Delta E_{\sigma}(\%)$ values are the percentage difference between both results, taking the $E_{\sigma}^{\mathrm{ac}}$ as the original number: $\left[\left|E_{\sigma}^{\mathrm{ac}}-E_{\sigma}^{\mathrm{dc}}\right| / E_{\sigma}^{\mathrm{ac}}\right] 100 \%$.

\begin{tabular}{llll}
\hline $\mathrm{M}$ & $E_{\sigma}^{\mathrm{dc}}(\mathrm{eV})$ & $E_{\sigma}^{\mathrm{ac}}(\mathrm{eV})$ & $\left|\Delta E_{\sigma}(\%)\right|$ \\
\hline $\mathrm{Si}$ & $0.75 \pm 0.03$ & $0.780 \pm 0.001$ & 3.8 \\
$\mathrm{Ti}$ & $0.77 \pm 0.01$ & $0.766 \pm 0.004$ & 0.5 \\
$\mathrm{Ge}$ & $0.75 \pm 0.02$ & $0.785 \pm 0.002$ & 4.5 \\
$\mathrm{Zr}$ & $0.71 \pm 0.02$ & $0.746 \pm 0.002$ & 4.8 \\
$\mathrm{Sn}$ & $0.74 \pm 0.01$ & $0.757 \pm 0.002$ & 2.2 \\
$\mathrm{Ce}$ & $0.78 \pm 0.01$ & $0.806 \pm 0.008$ & 3.2 \\
\hline
\end{tabular}

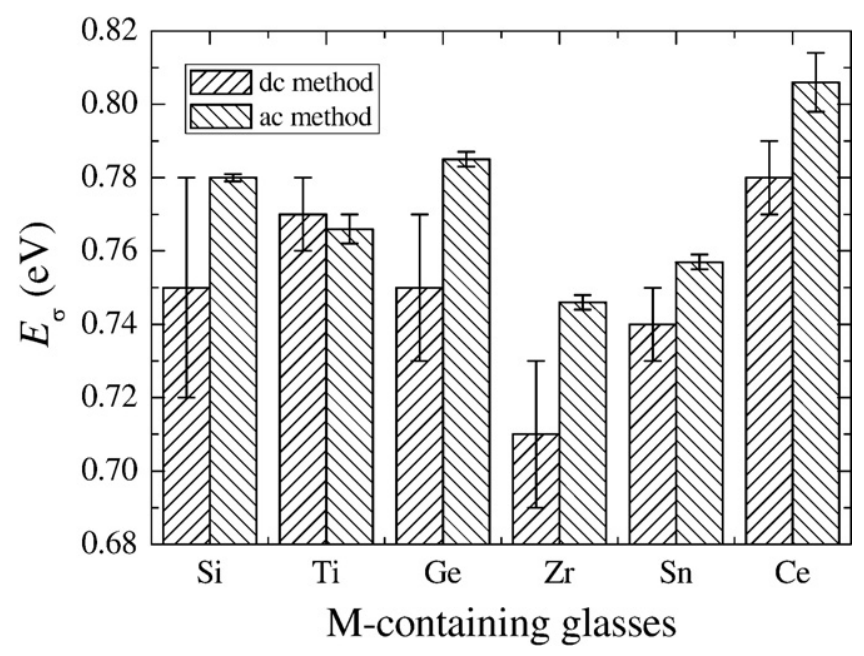

Fig. 8. Bar plot of the activation energy values reported in Table 1.

higher than $0.5 \mathrm{~s}$. The rate of this reaction gives rise to a reaction current, $i_{\mathrm{r}}=\mathrm{d} q_{\mathrm{r}} / \mathrm{d} t$, in addition to the polarization current, $i_{\mathrm{p}}$, that increases faster with increasing temperature due to the high electric field, and is also time dependent. The consequence of applying a high intensity electric field during longer time at higher temperatures is the removal of sodium from the cathodic surface and, depending on the electrode composition, the anodic adhesion [1,16]. The voltage pulses produced on the samples were short enough to avoid the sodium removal. The anodic adhesion was prevented by employing gold electrodes.

\section{Conclusion}

Electrical conductivity of silicate glasses had been determined by a $\mathrm{dc}$ method and their activation energies could be calculated. The results agree well with those obtained by the conventional ac method (impedance spectroscopy) and small differences are attributed to experimental uncertainties. In the present case, the dc method gave good results because:

1) High $V_{o}$ leads to measurable and accurate $I_{0}$ values that enable to calculate the electrical conductivity for several temperatures. Therefore, the activation energy could be calculated.

2) The effective charge carrier concentration was enough to give measurable $I_{0}$ values.

3) The time between two successive acquisitions were short enough to avoid the pile up and depletion of charge carriers at the electrodes. For the measured temperatures, the response of the external electrical circuit was shorter than the acquisition time.

4) There were no evidences of electrode reaction (Eq. (1)).

Therefore, the dc method employed showed to be a confident alternative for determining the conductivity and activation energy of silicate glasses, provided that some experimental conditions were satisfied.

In conclusion, the $I_{0}$ measured in the dc method is due to the same process of conduction that takes place in impedance spectroscopy, i.e., mainly cationic displacement by hopping over distances corresponding to their nearest neighbor separation.

\section{Acknowledgments}

The authors are grateful to Dr. Dante L. Chinaglia (DF-IGCE-UNESP) for experimental support, to MSc. Igor Fier and Dr. Lygia Walmsley (DF-IGCE-UNESP) for the assistance with the impedance measurements, and to Dr. René A. M. Alfaro and Dr. Makoto Yoshida (DF-IGCEUNESP) for helpful discussions. This research was supported by FAPESP (Grant Nos. 2008/07304-7 and 2008/07238-4). 


\section{References}

[1] E.C. Ziemath, V.D. Araújo, C.A. Escanhoela Jr., Compositional and structural changes at the anodic surface of thermally poled soda-lime float glass, J. Appl. Phys. 104 (2008) 054912.

[2] C.A. Escanhoela Jr., MSc. Dissertation, Universidade Estadual Paulista "Júlio de Mesquita Filho", Rio Claro-SP, Brazil, 2011.

[3] J.C. Dyre, P. Maass, B. Roling, D.L. Sidebottom, Fundamental questions relating to ion conduction in disordered solids, Rep. Prog. Phys. 72 (2009) 046501.

[4] M.L. Braunger, C.A. Escanhoela Jr., I. Fier, L. Walmsley, E.C. Ziemath, Electrical conductivity of silicate glasses with tetravalent cations substituting $\mathrm{Si}$, J. Non-Cryst. Solids 358 (2012) 2855-2861.

[5] M.L. Braunger, MSc. Dissertation, Universidade Estadual Paulista "Júlio de Mesquita Filho", Rio Claro-SP, Brazil, 2011.

[6] J. Vermeer, The electrical conduction of glass at high field strength, Physica 22 (1956) 1257-1268.

[7] J.P. Lacharme, J.O. Isard, Ion jump processes and high field conduction in glasses, J. Non-Cryst. Solids 27 (1978) 381-397.

[8] H.E. Taylor, The dielectric relaxation spectrum of glass, Trans. Faraday Soc. 52 (1956) 873-881.
[9] A. Doi, Y. Menjou, T. Ishikawa, Y. Abe, Generation and annihilation of positive and negative ion-depleted region in soda-lime silicate glass, J. Appl. Phys. 67 (1990) 691-697.

[10] R. Vaish, K.B.R. Varma, Dielectric properties of $\mathrm{Li}_{2} \mathrm{O}-3 \mathrm{~B}_{2} \mathrm{O}_{3}$ glasses, J. Appl. Phys. 106 (2009) 064106.

[11] R. Coelho, Physics of Dielectrics for Engineer, Elsevier, Amsterdam, 1979.

[12] A.M. Glass, $\mathrm{K}$. Nassau, Lithium ion conduction in rapidly quenched $\mathrm{Li}_{2} \mathrm{O}-\mathrm{Al}_{2} \mathrm{O}_{3}, \mathrm{Li}_{2} \mathrm{O}-$ $\mathrm{Ga}_{2} \mathrm{O}_{3}$, and $\mathrm{Li}_{2} \mathrm{O}-\mathrm{Bi}_{2} \mathrm{O}_{3}$ glasses, J. Appl. Phys. 67 (1980) 3756-3761.

[13] K.M. Shaju, S. Chandra, Silver ion conducting borate glass, Phys. Status Solidi B 181 (1994) 301-311

[14] D. Del Frate, S. Quilici, G. Spinolo, A. Vedda, High-temperature ac conductivity of amorphous $\mathrm{SiO}_{2}$ : fused silica and thin thermal films, Phys. Rev. B 59 (1999) 9741-9744.

[15] J.-L. Souquet, M.L.F. Nascimento, A.C.M. Rodrigues, Charge carrier concentration and mobility in alkali silicates, J. Chem. Phys. 132 (2010) 034704

[16] K.M. Knowles, A.T.J. van Helvoort, Anodic bonding, Int. Mater. Rev. 51 (2006) 273-311. 